\title{
1 Simulation-based comprehensive study of batch 2 effects in metabolomics studies
}

3 Miao $\mathbf{Y u}^{1+*}$, Anna Roszkowska ${ }^{1,2}$, Janusz Pawliszyn ${ }^{1^{*}}$

4 Department of Chemistry, University of Waterloo, 200 University Avenue West, Waterloo,

5 Ontario, N2L 3G1, Canada

62 Department of Pharmaceutical Chemistry, Medical University of Gdańsk, Gdańsk, Poland

7 *Corresponding author: Email: miao.yu@mssm.edu janusz@uwaterloo.ca Phone: +1-519-888-

8 4641. Fax: +1-519-746-0435

$9+$ current address: Department of Environmental Medicine and Public Health, Icahn School of

10 Medicine at Mount Sinai, New York, USA

11 Keywords: metabolomics, batch effects, in situ simulation 


\section{Abstract}

15 Batch effects will influence the interpretation of metabolomics data. In order to avoid misleading

16 results, batch effects should be corrected and normalized prior to statistical analysis.

17 Metabolomics studies are usually performed without targeted compounds (e.g., internal

18 standards) and it is a challenging task to validate batch effects correction methods. In addition,

19 statistical properties of metabolomics data are quite different from genomics data (where most of

20 the currently used batch correction methods have originated from). In this study, we firstly

21 analyzed already published metabolomics datasets so as to summarize and discuss their statistical

22 properties. Then, based on available datasets, we developed novel statistical properties-based in

23 silico simulations of metabolomics peaks' intensity data so as to analyze the influence of batch

24 effects on metabolomic data with the use of currently available batch correction strategies.

25 Overall, 252000 batch corrections on 14000 different in silico simulated datasets and related

26 differential analyses were performed in order to evaluate and validate various batch correction

27 methods. The obtained results indicate that log transformations strongly influence the

28 performance of all investigated batch correction methods. False positive rates increased after

29 application of batch correction methods with almost no improvement on true positive rates

30 among the analyzed batch correction methods. Hence, in metabolomic studies it is recommended

31 to implement preliminary experiments to simulate batch effects from real data in order to select

32 adequate batch correction method, based on a given distribution of peaks intensity. The

33 presented study is reproducible and related R package mzrtsim software can be found online

34 (https://github.com/yufree/mzrtsim). 


\section{Introduction}

40 Metabolomic studies focus on variations among metabolites in a given system with respect to 41 their exposure to different stimuli (Kusonmano et al., 2016), such as disease (Madsen et al., 42 2010, 2; Gonzalez-Riano et al., 2016) or environmental pollutants (Bundy et al., 2009; 43 Roszkowska et al., 2018). Gas chromatography coupled to mass spectrometry (GC-MS) and

44 liquid chromatography coupled to mass spectrometry (LC-MS) are major analytical methods extensively used to reveal changes in the metabolome (Alonso et al., 2015). However, in addition to the variables under study (experimental design), such as different therapies, and/or the use of different analytical methods, unwanted effects unrelated to biological variations among samples (e.g., batch effects) may also affect results in GC-MS/LC-MS based metabolomics analysis (Wehrens et al., 2016). Batch effects are commonly observed in highthroughput analytical methods, which generally involve one or multiple injection sequences during instrumental analysis (Goh et al., 2017; Pinto, 2017). Batch effects can stem from different factors. For instance, they can be related to known factors, such as sample injection order (Wang et al., 2013) or sample amount (Wu and Li, 2016), but could also be attributed to unknown factors unrelated to experimental design. Moreover, as most metabolomics studies are performed over long periods, the obtained results could be additionally influenced by different time points of sample collection, making data analysis and interpretation more complex (Zelena et al., 2009). Therefore, it is crucial to correct and normalize unwanted variables such as batch effects during the data analysis process so as to avoid irreproducible, incomplete, or misleading results.

60 Many research groups have been evaluating various batch effects correction strategies, but only 61 few have provided a detailed discussion on the influence of such effects on metabolomics data 62 (Wang et al., 2013; Hughes et al., 2014; Brunius et al., 2016; Jr et al., 2017; Ju et al., 2017; 63 Boysen et al., 2018). As shown in Scheme 1A, three theoretical types of batch effects could be 64 found in metabolomics datasets: I. monotonic (increasing or decreasing baseline), II. block 65 (different, but constant blocks/sequences), and III. mixed (with both monotone and block 66 changes observed simultaneously). These three types of batch effects could be corrected by a model considering such monotonic/block/mixed patterns. In high-throughput analytical methods 
same time. More important, such effects could be different for particular analytes due to the different physicochemical properties of the compounds under study or mass spectrometry insource reactions (Yu et al., 2017; Faber et al., 2014). To overcome this problem, a commonly applied approach entails application of internal standards; however, in untargeted metabolomics studies, the availability of internal standards is narrowed down to selected classes of metabolites; as such, fluctuations in signal intensities for other classes of analytes are unavoidable. As shown in Scheme 1B, if a single compound is used to normalize the data by its response, then bias would be introduced for other peaks possessing different batch effects. Nevertheless, some batch correction methods (Sysi-Aho et al., 2007; van der Kloet et al., 2009) still include the application of internal standards to normalize the whole data, which consequently might overcorrect peaks with different patterns of batch effects.

To date, several different methods have been developed to correct and normalize batch effects in metabolomics data. Kohl et al. compared nine different batch correction methods in an NMRbased metabolomics study, determining that the Quantile and Cubic-Spline Normalization method showed the best performance (Kohl et al., 2012). De Livera et al. reviewed seven normalized methods for removal of unwanted variations, and developed recommendations for selection of batch correction method with respect to the intended analytical application (Livera $e t$ al., 2015). Using the Shiny platform, Li et al. developed an online application for sixteen normalization methods, with results identifying the Variance Stabilization Normalization (VSN), Log Transformation, and Probabilistic Quotient Normalization (PQN) methods as most adequate for data normalization in metabolomics studies (Li et al., 2016). Wehrens et al. also compared several batch correction methods, concluding that when Quality Control (QC) samples and batch information were included in the models, general normalization methods also performed well (Wehrens et al., 2016). Overall, the appropriateness of a batch correction method for a given dataset is greatly dependent on whether the statistical model and assumption behind the correction method fit the real data, and as such, selection of an appropriate method should take these considerations into account.

97 The correction of batch effects in untargeted metabolomics is also challenging with respect to

98 validation of batch correction methods. In order to evaluate the performance of selected 
twenty-five correction methods, taking into account five criteria: intra-group variation among samples, the distribution of P-values from differential analysis, consistency of certain markers among different datasets, classification accuracy, and correspondence of reference data (Li et al., 2017). However, except for the last criteria, which actually uses the same routine as that used in targeted analysis, the proposed criteria scantily and no instinctively allow for selection of the most adequate correction method. Besides, each validation method was only based on ideal statistical properties or distributions, which might introduce bias when the intended data has a

107 different distribution or structure.

108 Moreover, if the batch effects for each peak are known, then validation and comparisons of different batch correction methods would be possible and easy. In this situation, direct counting

110 of peaks with real changes could be performed before and after the data were corrected.

111 However, such correction method cannot be applied when the research is designed to discover novel compounds (biomarkers) or to explore heterogeneity within the samples. In such cases, in silico simulations of such data would be a convenient tool to solve these issues. Simulation of 114 metabolomics data has been proposed in multiple studies (Mendes et al., 2005; Parsons et al., 115 2009; van der Kloet et al., 2009; Jr et al., 2017; Reisetter et al., 2017). However, similarly to 116 batch correction methods, previous simulation methods in metabolomics were developed from 117 data stemming from genomics microarray studies, and as such, did not consider the statistical 118 properties of MS-based metabolomics data, where multiple peaks from the same compound, such 119 as adducts, isotopologues, and fragment ions, would show high correlations. In this case, 120 metabolomics data have a hierarchical structure with correlations at both the peak and compound 121 level (Mahieu and Patti, 2017; Yu et al., 2019). In the case of metabolomics data, the 122 hierarchical structure indicates that all the peaks from the same compound are correlated with 123 each other, and that a given compound might also be correlated with other compounds. Hence, 124 peak level data also contains compound level correlations. Therefore, variances would be biased 125 on compounds with more peaks.

126 In this study, as a starting point, the statistical properties of GC-MS/LC-MS based data available 127 as already published datasets are discussed in order to elucidate what considerations should be 128 taken into account for in silico simulations. Next, we carried out in silico simulations of available 129 metabolomic datasets in order to evaluate various batch correction methods under different 
scenarios. In addition, the whole process developed as part of this study has been made reproducible and transparent by releasing all related functions and data processing code.

\section{Materials and methods}

133 Datasets

134 MTBLS28 (Mathé et al., 2014), MTBLS59 (Franceschi et al., 2012), MTBLS341 (Strehmel et 135 al., 2016), MTBLS351(Pedersen et al., 2016), MTBLS393 (Muhamadali et al., 2016) and the

136 faahKO data package (Saghatelian et al., 2004) were used to attain the statistical properties used

137 in the in silico simulations. In total, 14 related datasets were used to simulate 7 different

138 scenarios. These datasets are summarized in Table 1.

\section{Application of datasets for in silico simulation}

140 In untargeted metabolomics, GC-MS/LC-MS based data is represented by peaks occurring across

141 multiple samples. Statistical analysis of metabolomics data is thus performed based on the

142 experimental design. Given this, in silico simulations of such data should thus be commenced

143 with peaks attained from a single sample. The simulation could then be extended to multiple

144 samples based on the information contained in the real datasets.

145 Therefore, taking into account the experimental design for the selected datasets (Table 1), the

146 first step of this study encompassed calculations of average peak intensities across single

147 samples within particular groups. As presented in Figure 1, the log transformed intensity

148 distribution of detected peaks showed a left-skew pattern for Dataset 1, which contained 24

149 groups. Various patterns were observed for the other real datasets (Datasets 2-14); however,

150 none of these distributions could be simply expressed as normal distributions (see Figures S1-

151 S13). In order to simulate such peaks' intensities in silico, a Weibull distribution was applied to

152 the data, since such distribution provides the possibility for right- or left-skewed patterns (as

153 observed in real datasets), depending on the corresponding parameters, such as shape and scale.

154 In LC-MS analysis, each compound can generate multiple ions or peaks, such as isotopologues, 155 adducts, and neutral loss, on mass spectrometry (Yu et al., 2019). In GC-MS, specifically in EI 156 mode, each compound would generate multiple fragments along with molecular ions (Yu et al., 157 2017). In this study, the percentages of the detected compounds, expressed as the ratio between 158 the number of compounds and their corresponding peaks, were defined for real datasets and then 159 included into the in silico simulation. When such a parameter is lower than $50 \%$, more than two 
random peaks could come from a single compound. Moreover, according to previously reported work, 5-20\% of peaks could indicate either major variances or real compounds (Mahieu and Patti, 2017; Yu et al., 2019); as such, this assumption was taken into account during our simulation studies. We also assumed that correlated peaks from the same compound would not change the Weibull distribution for other peaks. In a simulation of the peaks' intensity, only a few peaks would be generated at first according to the Weibull distribution. For example, for a simulation of 1000 peaks (which are obtained from 100 compounds), only 100 "molecular ions" peaks would be generated at first according to the Weibull distribution. The other 900 peaks being simulated would account for adducts, neural loss, or fragmental ions based on resampling of those 100 "molecular ions" peaks, and would be weighted by a factor calculated from an exponential of normal distributed folds with mean 0 and standard deviation 1. Since peaks from the same compound would be correlated with one another, such a simulation should thus reflect the real ionization and/or fragmental process of compounds occurring during mass spectrometry analysis.

174 As a next step of the presented work, \%RSDs were calculated for samples in particular groups or 175 for biological replicates in each real dataset. Based on the obtained results for real datasets

176 (Figure 2 and Figure s14-s26), the applied \%RSDs distribution in in silico simulation could also 177 be a Weibull distribution.

178 After performing a simulation on single samples and then on biological replicates in one group, 179 the fold changes among different groups could be simulated. As presented in Figure 3 and 180 Figures S27-S39, most of the analyzed real datasets' fold changes among multiple samples in different groups followed an exponential distribution of normal distributed random errors. In this case, most peaks showed no changes among groups; however, few peaks presented large differences among groups. Since it is impossible to predict biological differences among groups, random peaks were selected to calculate a known fold change of intensity.

Based on the results from this analysis of multiple samples in real biological datasets, an in silico simulation study was carried out to generate 1000 peaks from two created biological groups (case and control), each containing 10 samples. The selected peaks from each real group showed different average intensities (according to the results obtained from the analysis of real samples), where peaks were influenced by different biological conditions (experimental design). Next, for the batch effects analysis, three previously mentioned types of batch effects, namely monotone, 
block, and mixed effects, were simulated in silico. As mentioned, for monotone batch effects, the

192 changed folds typically undergo an increasing/decreasing trend during the injection sequences.

193 For block batch effects, the changes would also follow an exponential distribution of normal

194 distributed random errors. To simulate mixed batch effects, both monotone changes and block

195 changes were considered.

196 In the simulations performed in this study, block batch effects affected 8, 5, and 7 samples,

197 whereas monotone batch effects were distributed across all samples. Influenced peaks from each

198 block batch showed different average intensities across multiple samples to simulate unwanted

199 batch effects. We considered that all peaks from the same compound were influenced by the

200 experimental design. However, batch effects were randomly assigned at the peak level since such

201 effects might also appear after the in-source ionization process. For peaks from a single sample,

202 the shape and scale for the Weibull distribution were simulated as 2 and 3. The parameters of the

203 Weibull distribution were selected based on the range of parameters observed in real datasets.

204 For the relative standard deviation (\%RSD), the shape and scale were set as 1 and 0.18 ,

205 respectively. Given the nature of such effects, analyses at the peak level, instead of the

206 compound level, were performed in evaluation studies of batch effect correction methods, which

207 are discussed later in this paper.

208 Next, based on the statistical probability of the available 14 real datasets, we simulated and

209 evaluated seven scenarios that are common in real research datasets (Table 2) in order to

210 evaluate and compare the performance of different batch effects correction strategies already

211 reported in the literature. Scenario 1 is the most common scenario applied for untargeted

212 analysis, with 100 compounds generating 1000 peaks, among which 100 peaks are influenced by

213 experimental design and 100 peaks are influenced by mixed batch effects. In scenario 2 ,

214 simulated compounds are almost independent of each other, a scenario similar to those observed

215 in targeted analysis approaches. Scenario 3 takes into account peak profiles, where half of the

216 peaks are influenced by the experimental design. This scenario is observed when the phenotype

217 shows obvious changes and untargeted analysis is performed to verify alterations at the

218 metabolite level. Scenario 4 reflects an experiment where half of the peaks are influenced by

219 mixed batch effects. Scenario 5 combines scenario 3 and scenario 4. Scenario 6 and scenario 7

220 concern the identification of differences between the two distinct types of batch effects:

221 monotone and block. Overall, each of the above-mentioned scenarios was simulated in silico 
1000 times. Simulation was performed on raw data and also on log transformed data for each scenario. In total, 252000 batch-corrected data were obtained on 14000 different simulated datasets to provide stable and reliable results for further discussion.

\section{Batch correction methods for simulated scenarios}

227 Finally, 17 batch correction methods, including autoscaling (van den Berg et al., 2006), pareto 228 scaling (van den Berg et al., 2006), range scaling (van den Berg et al., 2006), vast scaling (van den Berg et al., 2006), level scaling (van den Berg et al., 2006), total sum (De Livera et al., 2012), median (De Livera et al., 2012), mean (De Livera et al., 2012), Probabilistic Quotient Normalization (PQN) (Dieterle et al., 2006), Variance Stabilization Normalization (VSN) (Kohl et al., 2012), Quantile (Kohl et al., 2012), the robust spline normalization (RSN) from the lumi package (Lin et al., 2008), cyclic loss from the Limma package (Ballman et al., 2004), CUBIC Spline from the affy package (Workman et al., 2002), Surrogate Variable Analysis (SVA) (Leek and Storey, 2007, 2008), independent surrogate variable analysis (ISVA) (Teschendorff et al., 2011), and Principal component regression (PCR) (Yang et al., 2008), in addition to a no correction mode (where an uncorrected dataset was used as baseline), were selected in this study for a comparison of their performance in 7 simulated scenarios based on statistical properties from real datasets. The first 14 methods are general normalization methods, while the former 3 methods are batch correction methods based on linear models that assume latent batch effects could be treated as one variable in a linear model. In normalization methods, usually row-wise or column-wise adjustments are made without taking into account experimental design, whereas in

243 linear model based batch corrections, potential batch variables are considered and included in the 244 linear model to estimate the batch effects.

245 Statistical analysis was carried out in order to compare and contrast the 18 selected batch correction methods (17 methods and a no-method control), using computations of the true

247 positive rates and false positive rates of 1000 repeated simulations of each of the 7 scenarios 248 used in this study, with the false discovery rate controlled by Benjamini and Hochberg $(\mathrm{BH})$ 249 adjusted p-values. The cutoff of the BH adjusted p-value was set at 0.05, as typically used in 250 metabolomics differential analysis studies. The true positive rate is the ratio of the number of real 251 changed peaks found in real datasets (true positive) and the number of simulated changed peaks.

252 The false positive rate is the ratio between the number of false positive peaks and negative peaks. 
253 The simulation process and batch correction algorithm were run using the mzrtsim package and 254 script, which are listed in the supporting information.

\section{Results and discussion}

256

257

258

259

260

261

262

263

264

265

266

267

268

269

270

271

272

273

274

275

276

277

278

279

280

281

282

In total, 18 selected batch correction strategies were applied for each of the 7 scenarios, and their data compared and contrasted. The results of this evaluation study are discussed for each scenario in the next section.

\section{Comparison 1: Dependent peaks and Independent peaks}

In the first comparison, 18 batch correction methods were applied for Scenario 1 (dependent peaks) and Scenario 2 (independent peaks), where 100 simulated peaks out of 1000 peaks were real biological changes, and 100 simulated peaks could be influenced by the batch effects. The influences of existing relationships among peaks on batch effect correction were investigated and compared for each scenario. A comparison between these two scenarios was carried out to investigate whether the applied batch correction methods were influenced by the peaks' compound dependence.

As presented in Figure 4, when batch correction was performed on raw data, the 14 general batch correction methods (autoscaling, pareto scaling, range scaling, vast scaling, level scaling, total sum, median normalization, mean normalization, PQN, VSN, quantile, robust spline, cyclic loess, and CUBIC spline) showed similar true positive rates compared to data without correction (no correction), indicating that any of these 14 general methods could be effective when applied to cases similar to scenario 1 and scenario 2. On the other hand, the three linear models used in this study (SVA, ISVA, and PCR) were found to increase the false discovery rate, which is not a desired effect. However, once the data was log transformed, batch correction methods yielded a better performance as compared to that observed for raw data (Figure 4). When log-transformed data was used instead, a comparison of batch correction methods' performances between scenarios 1 and 2 revealed that the linear model-based correction methods provided larger false positive rates in dependent data (scenario observed in untargeted metabolomics study) in comparison to independent data. Therefore, the results suggest that in cases where metabolomics data from GC-MS/LC-MS based studies possess multiple peaks from the same compounds, such methods' false positive rate would be worse if the data were log transformed prior to correction as opposed to use in its raw format. 


\section{Comparison 2: experimental design dominated and batch effects dominated scenarios} In the second comparison, we set 100 compounds to generate 1000 peaks. In total, 3 unique datasets corresponding to 3 different scenarios were simulated for comparison. First, a dataset with 500 and 100 peaks changed by experimental design and batch effects, respectively, was simulated (scenario 3). This simulation took into account a scenario in which the resulting dataset characteristics would be primarily owed to the experimental design, and where most of the peaks could be used to separate biological groups. The second scenario considered in this comparison entailed a simulation with 100 and 500 peaks that were changed by experiment design and batch effects, respectively (scenario 4). Finally, a third scenario (scenario 5), combining properties of scenario 3 and scenario 4, and having 500 and 500 peaks changed by experiment design and batch effects, respectively, was included in this comparison. These three scenarios are commonly observed in real metabolomics datasets.

In this comparison, "no correction" data was treated as a baseline for each scenario. As presented in Figure 5, application of 17 batch correction methods on raw data revealed that scenarios 4 and 5 yielded much lower true positive rates and false positive rates (around 50\% and 60\%), respectively, when compared to treatment dominated data (around 80\%) in scenario 3. However, most of the batch correction methods used in these three scenarios made no improvements in the differential analysis, especially when applied to the raw data. Only linear model-based correction methods, such as SVA, yielded better performance on true positive rates for batch effects dominated data (scenario 4). However, the tradeoff of using such methods is incurring an increase in false positive rates compared with other batch correction methods. Interestingly, logtransformed data improved most of the correction methods' performance on true positive rates. In cases where studies are designed to yield high true positive rates, log transformation would be

\section{Comparison 3: influence of different types of batch effects}

309 The applied batch effects in the above-mentioned simulations were of mixed (monotone and 310 block) type only. However, in real datasets, observed batch effects may be mixed mode,

311 monotone, or block. Therefore, in the third comparison, the mixed batch effects were separated 312 into two different, not previously analyzed scenarios: one where the dataset was only affected by 313 monotone batch effects (scenario 6), and another one where only blocked batch effects affected 
314 the dataset (scenario 7). The performance of different batch correction methods on these two new

315 scenarios was then compared. All other parameters were the same as in scenario 1.

The obtained results indicate that the performances of all batch correction methods used in both

318 scenarios were similar; however, most of the analyzed methods had better performance towards monotone batch effects correction (Figure 6). Nevertheless, log transformation of the data still

320 had a strong influence on linear model-based batch correction methods, especially on false positive rates (see Figure 6). Therefore, the results presented in this comparison point to a pressing need for the development of new batch correction methods capable of addressing specific types of batch effects. Particularly in cases where the applied correction method is based on a linear model (such as the ones observed in scenarios 6 and 7), careful analysis of false positive rates should be carried out on the resulting data.

\section{Implication}

327 The results of the current study, which encompassed 252000 batch corrections on 14000

328 different simulated datasets, with subsequent differential analysis of the 18 available batch correction strategies on the generated data, show that none of the applied methods were fully useful for each scenario presented in this study, as all included batch correction methods were strongly influenced by the correction models and statistical properties of the data. Therefore, preliminary experiments should be implemented to simulate batch effects from real data. In this aspect, the proposed mzrtsim package could be used to simulate metabolomics data based on theoretical statistical distributions or on statistical distributions of real data. The obtained information could then be used to inform decisions regarding method selection, based on a given distribution of peaks intensity. However, since each peak would possess different batch effects, the application of pooled QC sampling would be a useful tool to control unwanted influences, ascertaining that only peaks with lower RSD\% in all pool QC samples are taken into account for further analysis (Dunn et al., 2012). Although the development of novel batch correction methods would be useful, particularly methods capable of addressing specific types of batch

341 effects, methods that ascertain whether it is worthwhile to apply batch corrections might be more meaningful for researchers. However, not all scenarios typically encountered in analytical studies were considered in this work. In the presented scenarios, typical of untargeted metabolomics, the 
344 analysis carried out as part of this work has been made reproducible as a script and could be

345 easily modified with different statistical properties or used directly to perform simulations from 346 real datasets.

\section{Conclusion}

Statistical properties and multipeak-based simulations reveal potential issues during data analysis, such as skewed distribution, different types of batch effects, and inner associations within data. Log transformation of datasets might be required as a preprocessing step in order to achieve better results. Corrections based on certain types of samples or peak(s) could cause overcorrection of other peaks. Further, traditional correction methods might increase the false positive rates of the datasets while not yielding much improvement with respect to true positive rates. While SVA, a linear model-based correction method, could be selected as a general batch correction method under different conditions after log transformation of the data, the method nonetheless still poses a risk of increasing false positive rates. This research was financially supported by Industrial Research Chair of the National Sciences and Engineering Research Council of Canada (NSERC-IRC).

\section{References}

Alonso,A. et al. (2015) Analytical Methods in Untargeted Metabolomics: State of the Art in 2015. Front. Bioeng. Biotechnol., 3.

Ballman,K.V. et al. (2004) Faster cyclic loess: normalizing RNA arrays via linear models. Bioinformatics, 20, 2778-2786.

van den Berg,R.A. et al. (2006) Centering, scaling, and transformations: improving the biological information content of metabolomics data. BMC Genomics, 7, 142.

Boysen,A.K. et al. (2018) Best-Matched Internal Standard Normalization in Liquid Chromatography-Mass Spectrometry Metabolomics Applied to Environmental Samples. Anal. Chem., 90, 1363-1369.

Brunius,C. et al. (2016) Large-scale untargeted LC-MS metabolomics data correction using between-batch feature alignment and cluster-based within-batch signal intensity drift correction. Metabolomics, 12, 173.

Bundy,J.G. et al. (2009) Environmental metabolomics: a critical review and future perspectives. Metabolomics, 5, 3.

De Livera,A.M. et al. (2012) Normalizing and Integrating Metabolomics Data. Anal. Chem., 84, 10768-10776.

Dieterle,F. et al. (2006) Probabilistic Quotient Normalization as Robust Method to Account for Dilution of Complex Biological Mixtures. Application in 1H NMR Metabonomics. Anal. Chem., 78, 4281-4290.

Dunn,W.B. et al. (2012) The importance of experimental design and QC samples in large-scale and MS-driven untargeted metabolomic studies of humans. Bioanalysis, 4, 2249-2264. 
374

375

376

377

378

379

380

381

382

383

384

385

386

387

388

389

390

391

392

393

394

395

396

397

398

399

400

401

402

403

404

405

406

407

408

409

410

411

412

413

414

415

416

417

418

419

420

421

422

423

424

Faber,H. et al. (2014) Electrochemistry/mass spectrometry as a tool in metabolism studies-A review. Anal. Chim. Acta, 834, 9-21.

Franceschi,P. et al. (2012) A benchmark spike-in data set for biomarker identification in metabolomics. J. Chemom., 26, 16-24.

Goh,W.W.B. et al. (2017) Why Batch Effects Matter in Omics Data, and How to Avoid Them. Trends Biotechnol., 35, 498-507.

Gonzalez-Riano,C. et al. (2016) Metabolomics studies in brain tissue: A review. J. Pharm. Biomed. Anal., 130, 141-168.

Hughes, G. et al. (2014) MSPrep-Summarization, normalization and diagnostics for processing of mass spectrometry-based metabolomic data. Bioinformatics, 30, 133-134.

Jr,S.S. et al. (2017) RRmix: A method for simultaneous batch effect correction and analysis of metabolomics data in the absence of internal standards. PLOS ONE, 12, e0179530.

Ju,J.H. et al. (2017) An independent component analysis confounding factor correction framework for identifying broad impact expression quantitative trait loci. PLoS Comput. Biol., 13.

van der Kloet,F.M. et al. (2009) Analytical Error Reduction Using Single Point Calibration for Accurate and Precise Metabolomic Phenotyping. J. Proteome Res., 8, 5132-5141.

Kohl,S.M. et al. (2012) State-of-the art data normalization methods improve NMR-based metabolomic analysis. Metabolomics, 8, 146-160.

Kusonmano,K. et al. (2016) Informatics for Metabolomics. In, Translational Biomedical Informatics, Advances in Experimental Medicine and Biology. Springer, Singapore, pp. $91-115$.

Leek,J.T. and Storey,J.D. (2008) A general framework for multiple testing dependence. Proc. Natl. Acad. Sci., 105, 18718-18723.

Leek,J.T. and Storey,J.D. (2007) Capturing Heterogeneity in Gene Expression Studies by Surrogate Variable Analysis. PLOS Genet, 3, e161.

Li,B. et al. (2017) NOREVA: normalization and evaluation of MS-based metabolomics data. Nucleic Acids Res., 45, W162-W170.

Li,L. et al. (2016) Comprehensive investigation of tobacco leaves during natural early senescence via multi-platform metabolomics analyses. Sci. Rep., 6.

Lin,S.M. et al. (2008) Model-based variance-stabilizing transformation for lllumina microarray data. Nucleic Acids Res., 36, e11-e11.

Livera,A.M.D. et al. (2015) Statistical Methods for Handling Unwanted Variation in Metabolomics Data. Anal. Chem., 87, 3606-3615.

Madsen, R. et al. (2010) Chemometrics in metabolomics-A review in human disease diagnosis. Anal. Chim. Acta, 659, 23-33.

Mahieu,N.G. and Patti,G.J. (2017) Systems-Level Annotation of a Metabolomics Data Set Reduces 25 $\square 000$ Features to Fewer than 1000 Unique Metabolites. Anal. Chem., 89, 10397-10406.

Mathé,E.A. et al. (2014) Noninvasive urinary metabolomic profiling identifies diagnostic and prognostic markers in lung cancer. Cancer Res., 74, 3259-3270.

Mendes,P. et al. (2005) Modelling and simulation for metabolomics data analysis. Biochem. Soc. Trans., 33, 1427-1429.

Muhamadali,H. et al. (2016) Metabolomic analysis of riboswitch containing E. coli recombinant expression system. Mol. Biosyst., 12, 350-361.

Parsons,H.M. et al. (2009) Spectral relative standard deviation: a practical benchmark in metabolomics. Analyst, 134, 478-485.

Pedersen,H.K. et al. (2016) Human gut microbes impact host serum metabolome and insulin sensitivity. Nature, 535, 376-381.

Pinto,R.C. (2017) Chemometrics Methods and Strategies in Metabolomics. In, Metabolomics: From Fundamentals to Clinical Applications, Advances in Experimental Medicine and 
Biology. Springer, Cham, pp. 163-190.

Reisetter,A.C. et al. (2017) Mixture model normalization for non-targeted gas chromatography/mass spectrometry metabolomics data. BMC Bioinformatics, 18.

Roszkowska,A. et al. (2018) Metabolome Profiling of Fish Muscle Tissue Exposed to Benzo[a]pyrene Using in Vivo Solid-Phase Microextraction. Environ. Sci. Technol. Lett., 5, 431-435.

Saghatelian,A. et al. (2004) Assignment of Endogenous Substrates to Enzymes by Global Metabolite Profiling. Biochemistry, 43, 14332-14339.

Strehmel,N. et al. (2016) Piriformospora indica Stimulates Root Metabolism of Arabidopsis thaliana. Int. J. Mol. Sci., 17, 1091.

Sysi-Aho,M. et al. (2007) Normalization method for metabolomics data using optimal selection of multiple internal standards. BMC Bioinformatics, 8, 93.

Teschendorff,A.E. et al. (2011) Independent surrogate variable analysis to deconvolve confounding factors in large-scale microarray profiling studies. Bioinformatics, 27, 14961505.

Wang,S.-Y. et al. (2013) Batch Normalizer: A Fast Total Abundance Regression Calibration Method to Simultaneously Adjust Batch and Injection Order Effects in Liquid Chromatography/Time-of-Flight Mass Spectrometry-Based Metabolomics Data and Comparison with Current Calibration Methods. Anal. Chem., 85, 1037-1046.

Wehrens, R. et al. (2016) Improved batch correction in untargeted MS-based metabolomics. Metabolomics, 12, 88.

Workman,C. et al. (2002) A new non-linear normalization method for reducing variability in DNA microarray experiments. Genome Biol., 3, research0048.1-research0048.16.

Wu,Y. and Li,L. (2016) Sample normalization methods in quantitative metabolomics. J. Chromatogr. A, 1430, 80-95.

Yang,H. et al. (2008) Randomization in Laboratory Procedure Is Key to Obtaining Reproducible Microarray Results. PLOS ONE, 3, e3724.

Yu,M. et al. (2017) Evaluation and reduction of the analytical uncertainties in GC-MS analysis using a boundary regression model. Talanta, 164, 141-147.

Yu,M. et al. (2019) Structure/reaction directed analysis for LC-MS based untargeted analysis. Anal. Chim. Acta, 1050, 16-24.

Zelena,E. et al. (2009) Development of a Robust and Repeatable UPLC-MS Method for the Long-Term Metabolomic Study of Human Serum. Anal. Chem., 81, 1357-1364. 
460 Table 1. Summary of the datasets used in this study.

461

\begin{tabular}{|c|c|c|c|c|c|c|c|c|}
\hline ID & Study & $\begin{array}{l}\text { Analytica } \\
1 \text { method }\end{array}$ & Organism & $\begin{array}{l}\text { Experime } \\
\text { ntal } \\
\text { Factors }\end{array}$ & $\begin{array}{l}\text { Number } \\
\text { of } \\
\text { samples }\end{array}$ & $\begin{array}{l}\text { Number } \\
\text { of groups }\end{array}$ & $\begin{array}{l}\text { Number } \\
\text { of } \\
\text { features }\end{array}$ & $\begin{array}{l}\text { Referenc } \\
\text { es }\end{array}$ \\
\hline Dataset 1 & $\begin{array}{l}\text { MTBLS2 } \\
8\end{array}$ & $\begin{array}{l}\text { UPLC-Q- } \\
\text { TOF } \\
\text { Positive }\end{array}$ & $\begin{array}{l}\text { Homo } \\
\text { sapiens }\end{array}$ & $\begin{array}{l}\text { Gender\& } \\
\text { Race\&S } \\
\text { moking\& } \\
\text { Sample } \\
\text { Type } \\
\text { Gender\& } \\
\text { Race\&S } \\
\text { moking\& }\end{array}$ & 1005 & 24 & 1807 & $\begin{array}{l}\text { (Mathé et } \\
\text { al., 2014) }\end{array}$ \\
\hline Dataset2 & $\begin{array}{l}\text { MTBLS2 } \\
8\end{array}$ & $\begin{array}{l}\text { TOF } \\
\text { Negative } \\
\text { UPLC-Q- } \\
\text { TOF }\end{array}$ & $\begin{array}{l}\text { Homo } \\
\text { sapiens } \\
\text { Malus } \\
\text { domestic }\end{array}$ & $\begin{array}{l}\text { Sample } \\
\text { Type }\end{array}$ & 1005 & 24 & 1359 & $\begin{array}{l}\text { (Mathé et } \\
\text { al., 2014) } \\
\text { (Frances } \\
\text { chi et al., }\end{array}$ \\
\hline Dataset3 & MTBLS5 & $\begin{array}{l}\text { Positive } \\
\text { UPLC-Q- } \\
\text { TOF }\end{array}$ & $\begin{array}{l}\text { a } \\
\text { Malus } \\
\text { domestic }\end{array}$ & Spiking & 40 & 4 & 1632 & $\begin{array}{l}\text { 2012) } \\
\text { (Frances } \\
\text { chi et al., }\end{array}$ \\
\hline Dataset4 & 9 & Negative & $\begin{array}{l}\text { a } \\
\text { Arabidop }\end{array}$ & Spiking & 40 & 4 & 995 & 2012) \\
\hline Dataset5 & $\begin{array}{l}\text { MTBLS3 } \\
41\end{array}$ & $\begin{array}{l}\text { UPLC-Q- } \\
\text { TOF } \\
\text { Positive }\end{array}$ & $\begin{array}{l}\text { sis } \\
\text { thaliana } \\
\text { root }\end{array}$ & $\begin{array}{l}\text { Replicate } \\
\text { \&Treatm } \\
\text { ent }\end{array}$ & 20 & 4 & 3310 & $\begin{array}{l}\text { (Strehmel } \\
\text { et al., } \\
\text { 2016) }\end{array}$ \\
\hline Dataset6 & $\begin{array}{l}\text { MTBLS3 } \\
41\end{array}$ & $\begin{array}{l}\text { UPLC-Q- } \\
\text { TOF } \\
\text { Negative }\end{array}$ & $\begin{array}{l}\text { Arabidop } \\
\text { sis } \\
\text { thaliana } \\
\text { root }\end{array}$ & $\begin{array}{l}\text { Replicate } \\
\text { \&Treatm } \\
\text { ent }\end{array}$ & 20 & 4 & 2542 & $\begin{array}{l}\text { (Strehmel } \\
\text { et al., } \\
2016 \text { ) }\end{array}$ \\
\hline Dataset7 & $\begin{array}{l}\text { MTBLS3 } \\
41\end{array}$ & $\begin{array}{l}\text { UPLC-Q- } \\
\text { TOF } \\
\text { Positive }\end{array}$ & $\begin{array}{l}\text { Arabidop } \\
\text { sis } \\
\text { thaliana } \\
\text { leaf }\end{array}$ & $\begin{array}{l}\text { Replicate } \\
\text { \&Treatm } \\
\text { ent }\end{array}$ & 18 & 4 & 1723 & $\begin{array}{l}\text { (Strehmel } \\
\text { et al., } \\
2016)\end{array}$ \\
\hline Dataset8 & $\begin{array}{l}\text { MTBLS3 } \\
41\end{array}$ & $\begin{array}{l}\text { UPLC-Q- } \\
\text { TOF } \\
\text { Negative }\end{array}$ & $\begin{array}{l}\text { Arabidop } \\
\text { sis } \\
\text { thaliana } \\
\text { leaf }\end{array}$ & $\begin{array}{l}\text { Replicate } \\
\text { \&Treatm } \\
\text { ent }\end{array}$ & 18 & 4 & 1359 & $\begin{array}{l}\text { (Strehmel } \\
\text { et al., } \\
2016)\end{array}$ \\
\hline
\end{tabular}




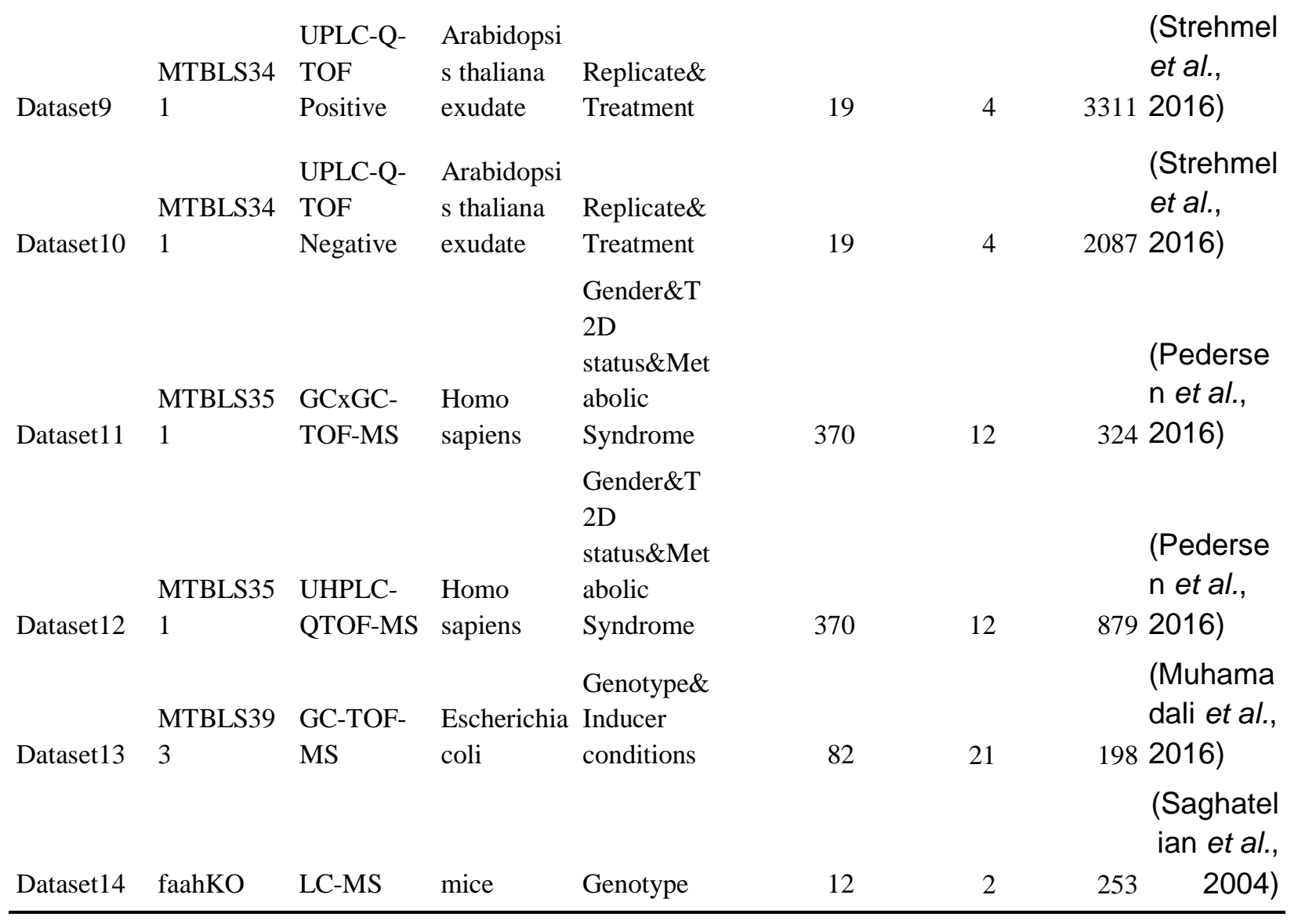


Table 2. Parameters for different simulation scenarios

464

\begin{tabular}{lllll}
\hline & compounds\# & changed peaks & Batch peaks & Batch type \\
\hline Scenario 1 & 100 & 100 & 100 & mixed \\
Scenario 2 & 900 & 100 & 100 & mixed \\
Scenario 3 & 100 & 500 & 100 & mixed \\
Scenario 4 & 100 & 100 & 500 & mixed \\
Scenario 5 & 100 & 500 & 500 & mixed \\
Scenario 6 & 100 & 100 & 100 & monotone \\
Scenario 7 & 100 & 100 & 100 & block \\
\hline
\end{tabular}




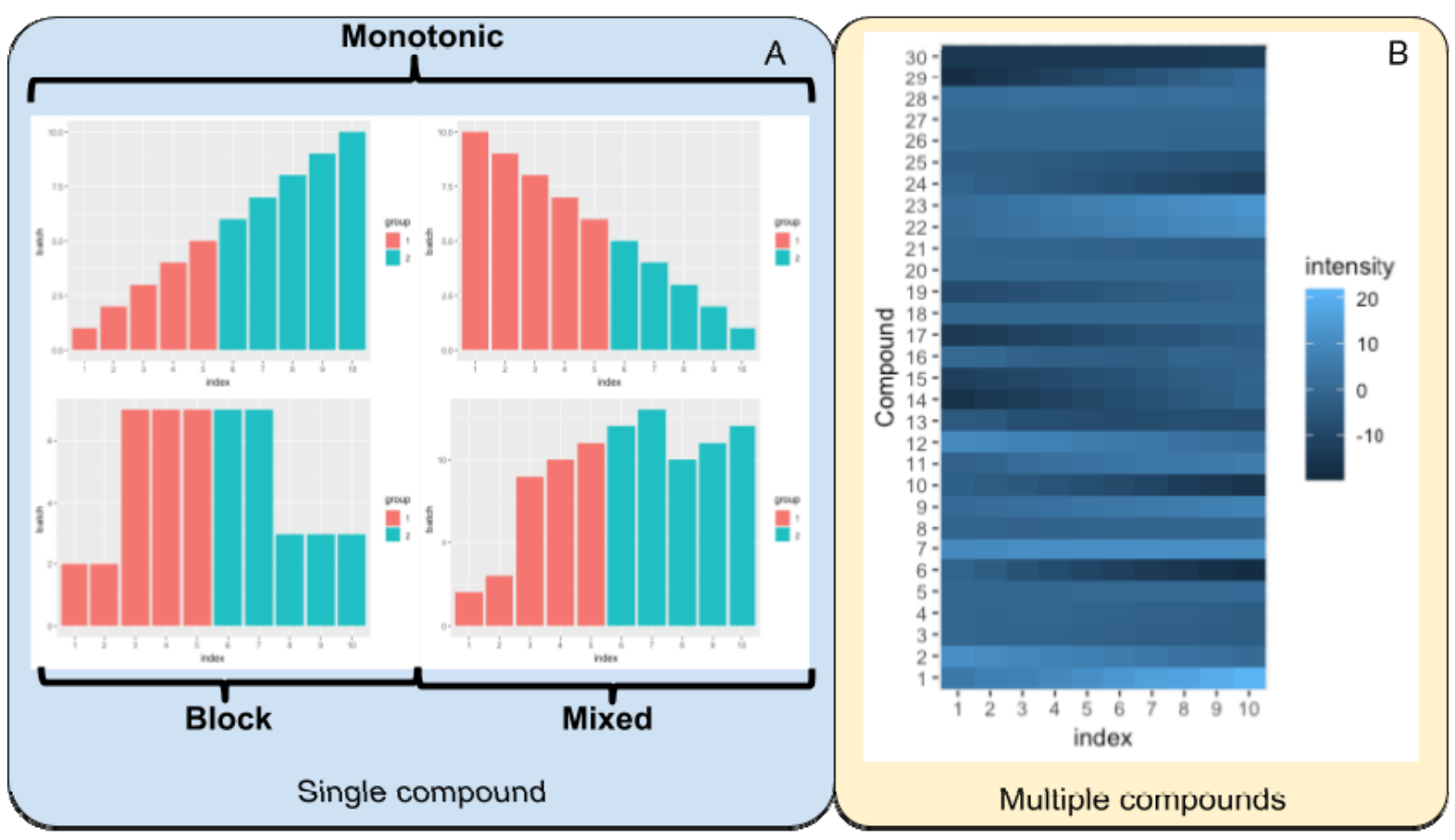

468 Scheme 1. Batch effects in metabolomics studies. (A) Three types of batch effects occurring on a 469 single compound. (B) Demonstration of batch effects on multiple compounds with different 470 types of batch effects observed in high-throughput analysis. 

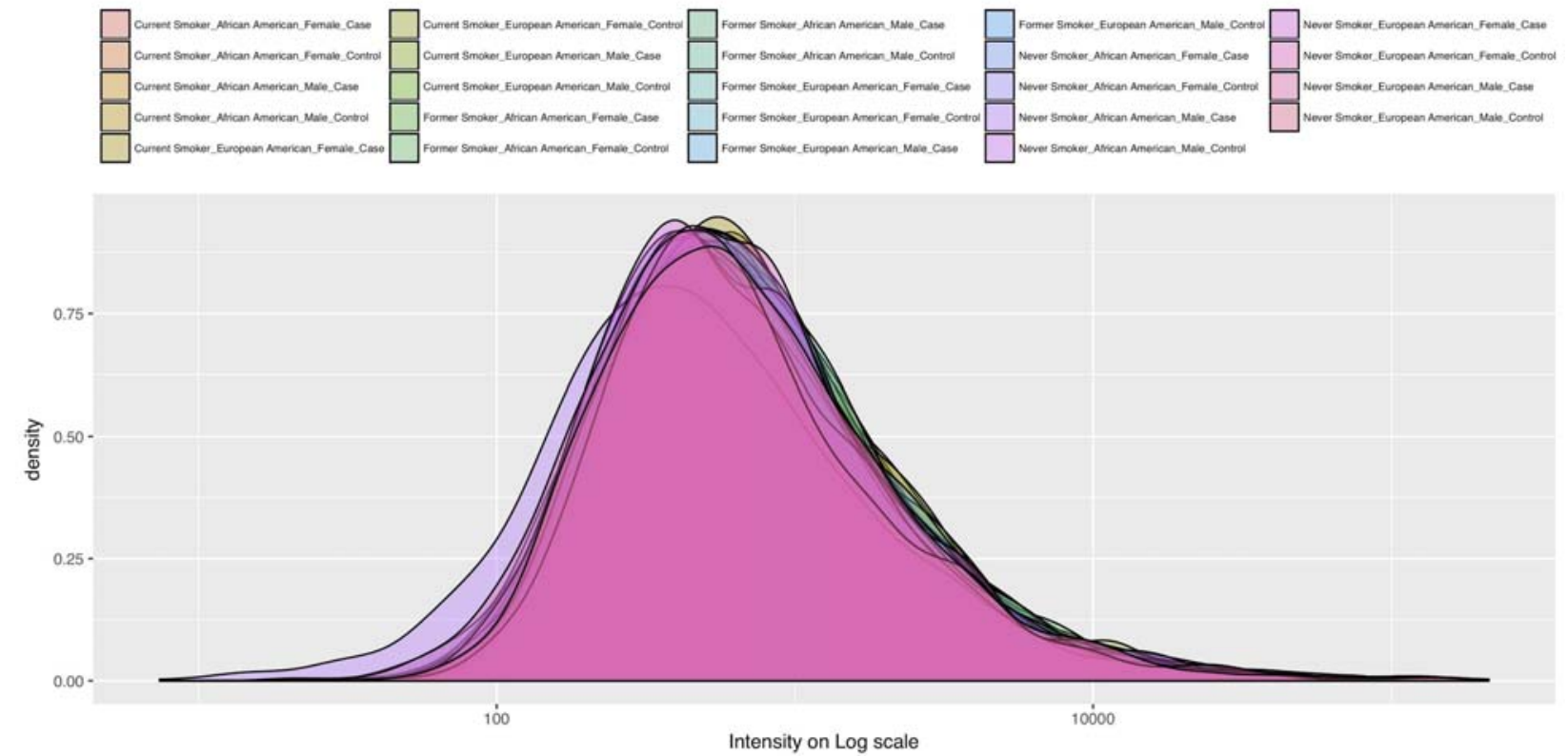

Figure 1. Distribution of real peaks' intensities on the Log scale for Dataset 1. 


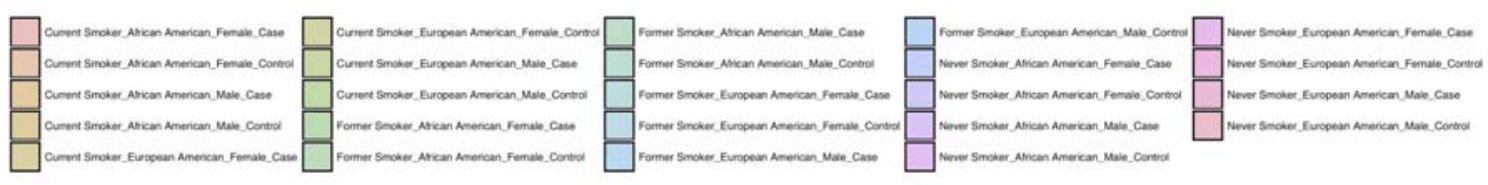




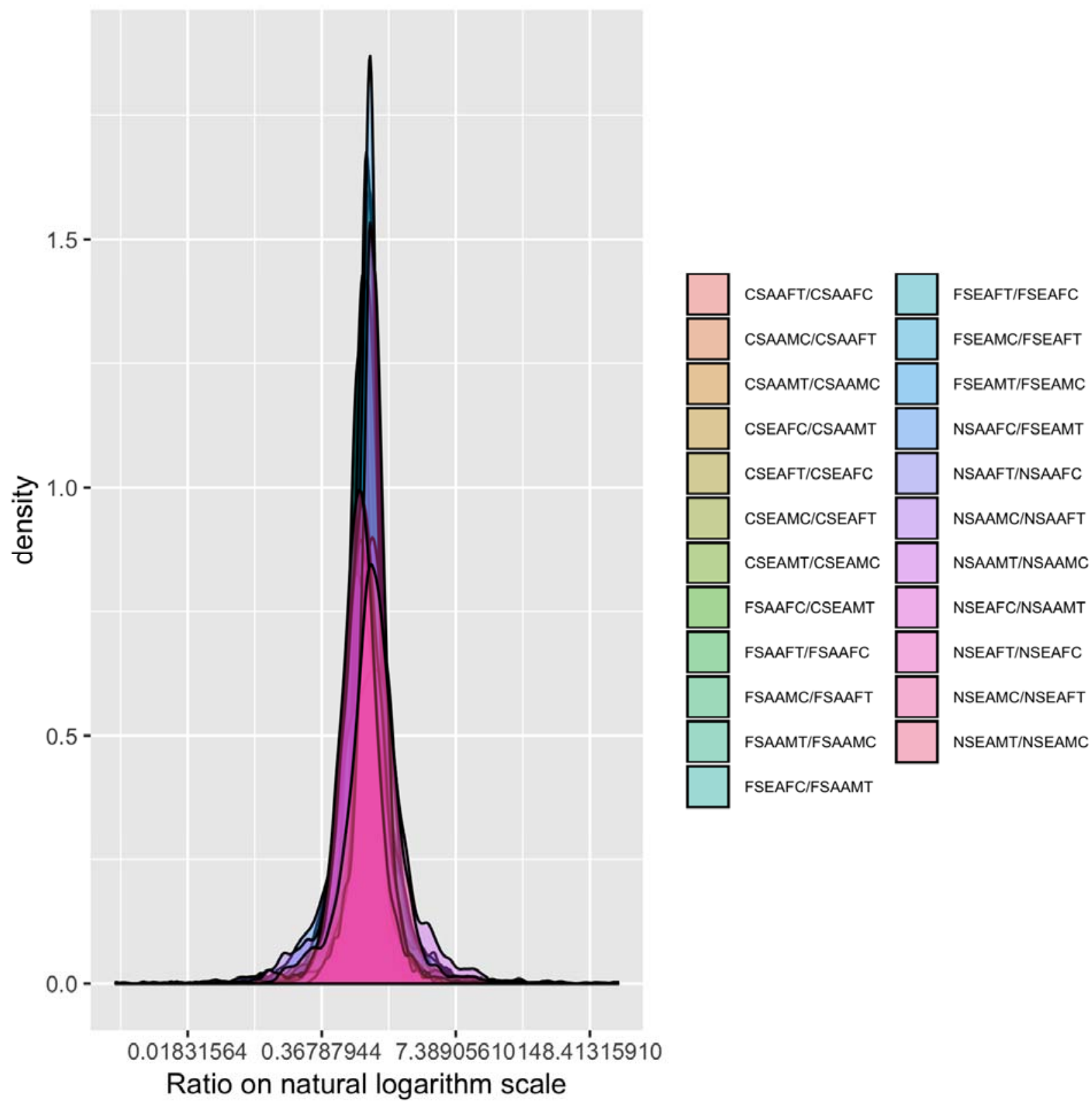

Figure 3. Distributions of ratios of group averages for Dataset 1. 

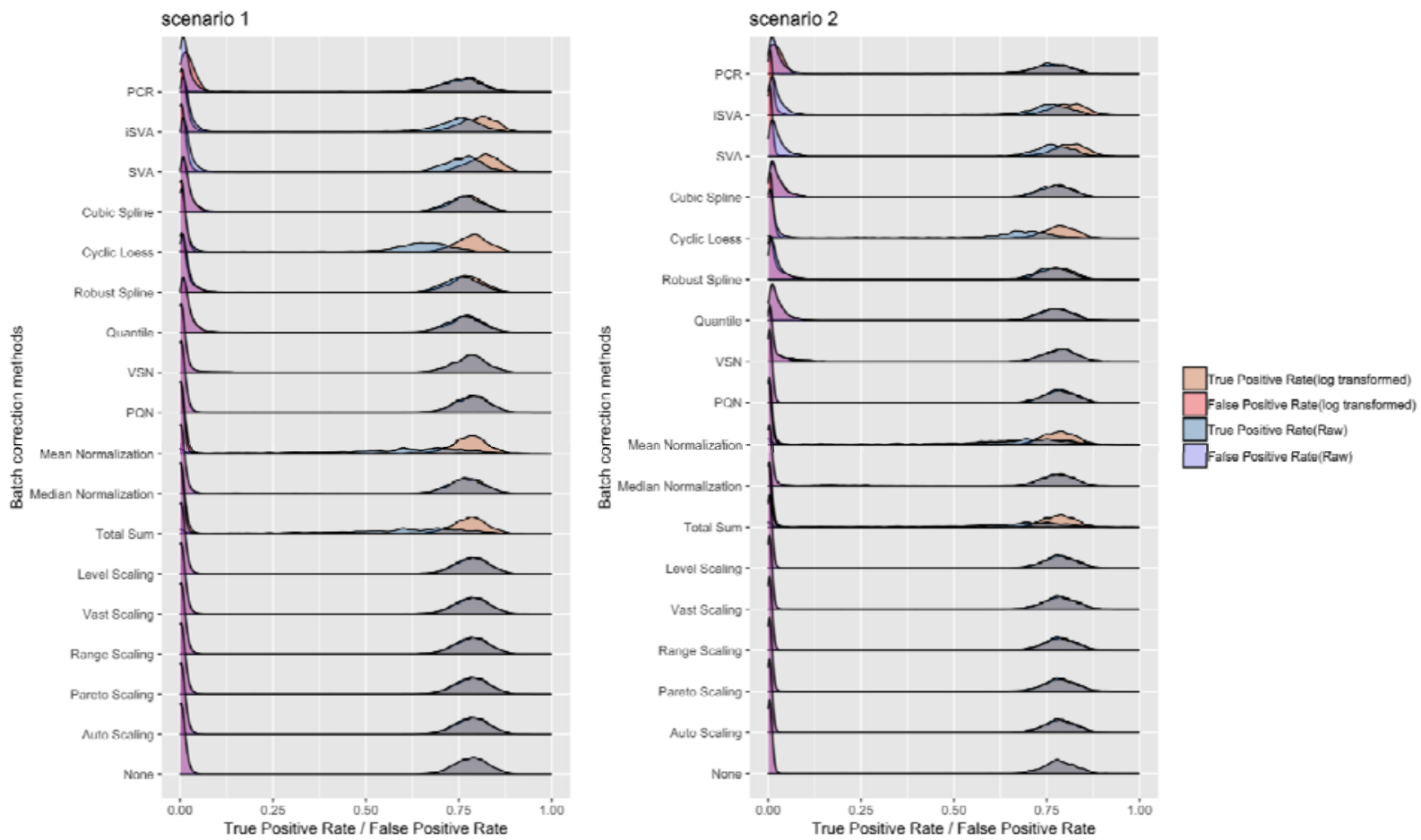

Figure 4. Ridges plot of true positive rates and false positive rates of 1000 times simulated data when the $\mathrm{BH}$ adjusted $\mathrm{p}$-value cutoff is 0.05 for scenario 1 and scenario 2 , and their log 

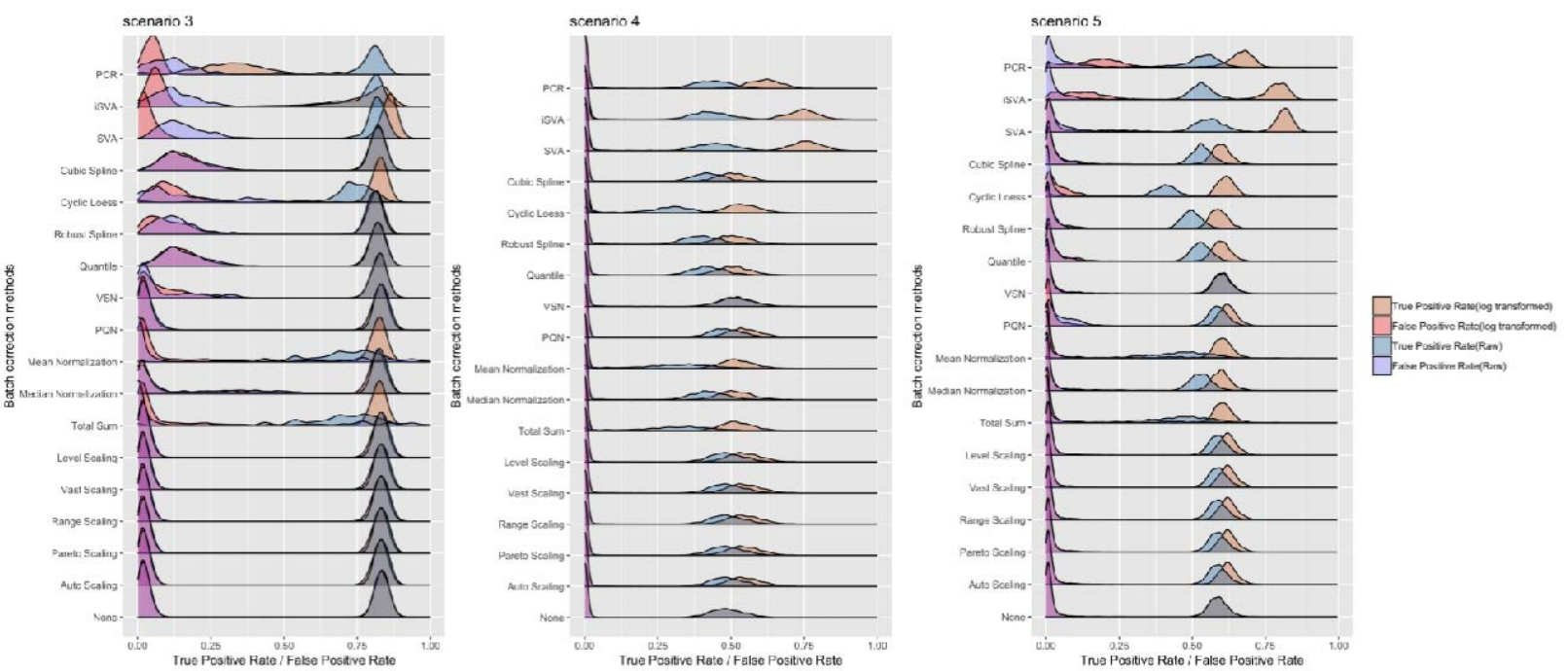

Figure 5. Ridges plot of true positive rates and false positive rates of 1000-times simulated data.

490 The $\mathrm{BH}$ adjusted p-value cutoff was set at 0.05 for scenario 3,4 and 5 , and their log

491 transformed data. 

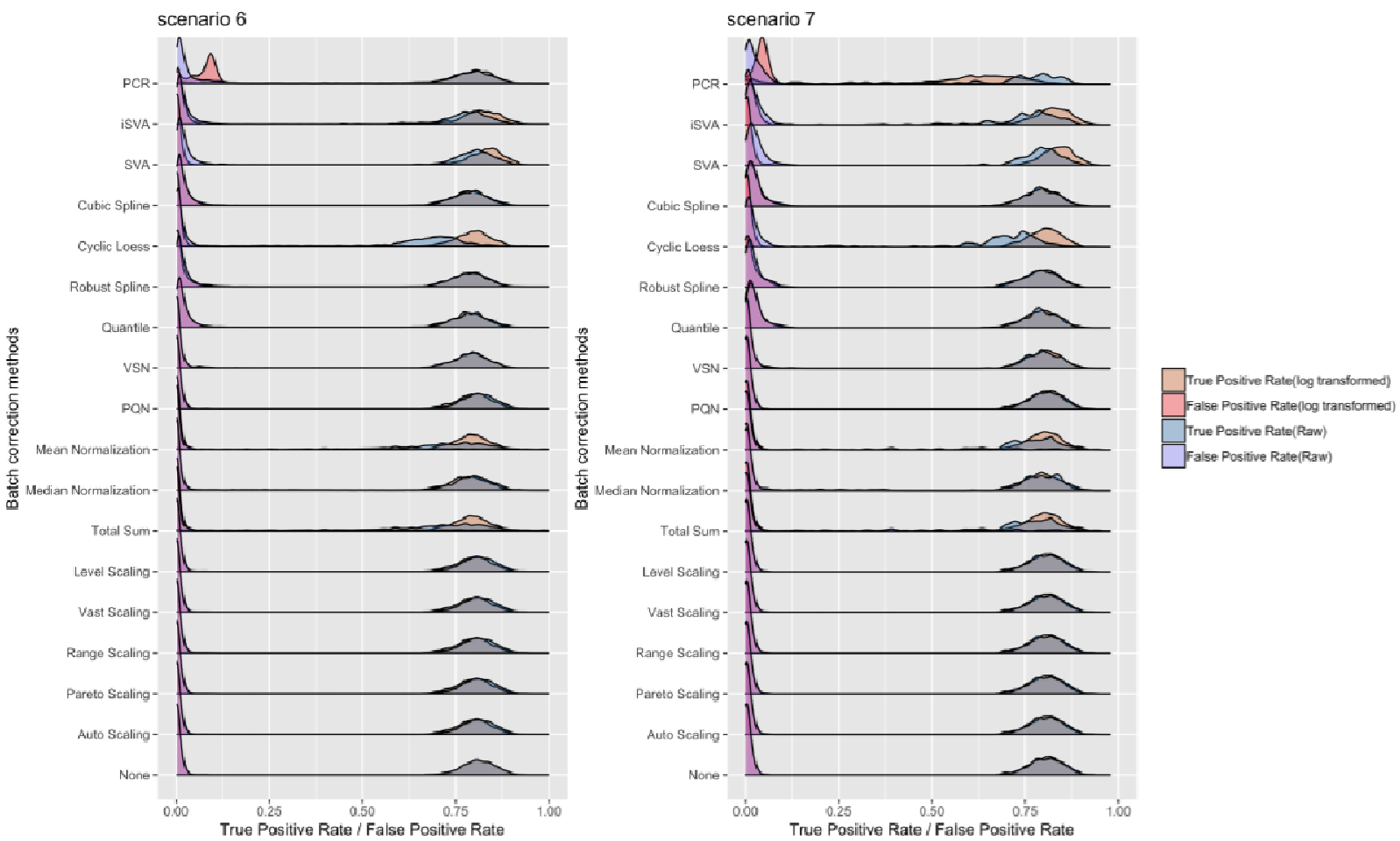

Figure 6. Ridges plot of true positive rates and false positive rates of 1000 times simulated data when the $\mathrm{BH}$ adjusted $\mathrm{p}$ value cutoff is 0.05 for scenarios 6 and 7 , and their log transformed data. 